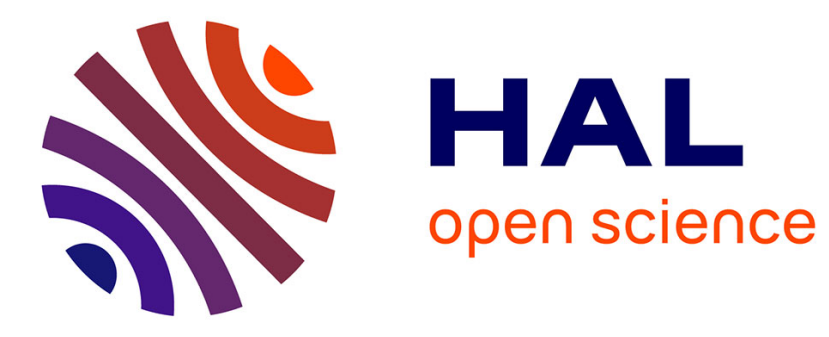

\title{
Magnitude and complex based diffusion signal reconstruction
}

Marco Pizzolato, Aurobrata Ghosh, Timothé Boutelier, Rachid Deriche

\section{To cite this version:}

Marco Pizzolato, Aurobrata Ghosh, Timothé Boutelier, Rachid Deriche. Magnitude and complex based diffusion signal reconstruction. CDMRI'14, Sep 2014, Boston, United States. 10.1007/978-3319- . hal-01095127

\author{
HAL Id: hal-01095127 \\ https://hal.inria.fr/hal-01095127
}

Submitted on 15 Dec 2014

HAL is a multi-disciplinary open access archive for the deposit and dissemination of scientific research documents, whether they are published or not. The documents may come from teaching and research institutions in France or abroad, or from public or private research centers.
L'archive ouverte pluridisciplinaire HAL, est destinée au dépôt et à la diffusion de documents scientifiques de niveau recherche, publiés ou non, émanant des établissements d'enseignement et de recherche français ou étrangers, des laboratoires publics ou privés. 


\title{
Magnitude and complex based diffusion signal reconstruction
}

\author{
Marco Pizzolato, Aurobrata Ghosh, Timothé Boutelier, and Rachid Deriche
}

\begin{abstract}
In Diffusion Weighted Magnetic Resonance Imaging (DW-MRI) the modeling of the magnitude signal is complicated by the Rician distribution of the noise. It is well known that when dealing instead with the complex valued signal, the real and imaginary parts are affected by Gaussian distributed noise and their modeling can thus benefit from any estimation technique suitable for this noise distribution. We present a quantitative analysis of the difference between the modeling of the magnitude diffusion signal and the modeling in the complex domain. The noisy complex and magnitude diffusion signals are obtained for a physically realistic scenario in a region close to a restricting boundary. These signals are then fitted with the Simple Harmonic Oscillator based Reconstruction and Estimation (SHORE) bases and the reconstruction performances are quantitatively compared. The noisy magnitude signal is also fitted by taking into account the Rician distribution of the noise via the integration of a Maximum Likelihood Estimator (MLE) in the SHORE. We discuss the performance of the reconstructions as function of the Signal to Noise Ratio (SNR) and the sampling resolution of the diffusion signal. We show that fitting in the complex domain generally allows for quantitatively better signal reconstruction, also with a poor SNR, provided that the sampling resolution of the signal is adequate. This applies also when the reconstruction is compared to the one performed on the magnitude via the MLE. ${ }^{1}$
\end{abstract}

Marco Pizzolato

Athena, Inria Sophia Antipolis - Méditerranée, France, e-mail: marco.pizzolato@inria.fr

Aurobrata Ghosh

Athena, Inria Sophia Antipolis - Méditerranée, France, e-mail: aurobrata.ghosh@gmail.com

Timothé Boutelier

Olea Medical, La Ciotat, France, e-mail: timothe.boutelier@olea-medical.com

Rachid Deriche

Athena, Inria Sophia Antipolis - Méditerranée, France, e-mail: rachid.deriche@inria.fr

1 The final publication will be available at http://link.springer.com/book/10.1007/978-3-319-

11182-7, (C) Springer-Verlag 2015. 


\section{Introduction}

Diffusion Weighted magnetic resonance imaging (DW-MRI) measures the signal attenuation due to the loss of spin phase coherence caused by particles subject to Brownian motion. DW-MRI thus is inherently a low Signal to Noise Ratio (SNR) technique. Indeed, increasing the diffusion-weighting measured by the b-value or decreasing the voxel size can further reduce the SNR, causing the signal to be close to the background noise level [7]. This is particularly problematic when characterizing the non-Gaussianity of the diffusion signal profile, associated to restricted diffusion, since a high b-value is generally required [1]. Indeed a nonlinear dependence between the log-transformed DW intensity and the diffusion-weighting (b-value), as the sole result of noise, has been previously reported [7]. This can lead to misinterpretations of the underlying diffusion process which, for instance, can be erroneously thought as restricted, thus ascribing to the tissue more structural complexity than exists. However, one of the major sources of error in magnetic resonance imaging (MRI), and even more accentuated in DW-MRI due to the inherent low SNR, is the noise.

In MRI the signal, acquired for each coil in quadrature, is complex with an additive thermal noise that can be considered to be derived from a bivariate normal distribution $N(0, \sigma)$ [6]. However, when the magnitude of the complex signal is computed, the noise becomes Rician distributed [5] and a not-negligible noise floor, the minimum signal measurable, appears. The bias introduced by the Rician distribution leads to the distortion of estimated quantitative diffusion parameters. For instance, the noise floor causes the DW signal to be overestimated, leading to underestimation of the Apparent Diffusion Coefficient (ADC) [4]. Other noise-related issues have also been reported, such as orientationally dependent deviation from Gaussianity of the ADC profile, underestimation of diffusion anisotropy indices and correlation between mean diffusivity and diffusion anisotropy [7]. Hence, denoising is essential in DW-MRI.

To properly denoise magnitude signals, the noise distribution should be taken into account. However, the distribution of the noise affecting magnitude DW images (DWIs) changes depending on the number of coils used for the acquisition and on the employed reconstruction method. For instance, when magnitude images are obtained via the sum-of-squares reconstruction from multiple coils, the noise follows a non-central $\chi$ distribution [3]. Nevertheless, the complex diffusion signal is still affected by noise with a Gaussian distribution, which can be exploited via any Gaussian-based denoising technique or fitting procedure.

In this paper we analyze the theoretical performance gain given by considering the complex signal instead of just using the magnitude. The complex signal is synthetically generated, according to [9], by considering a voxel located close to a boundary, where the underlying diffusion process is restricted. In fact, by exploiting the asymmetry, due to the presence of the boundary, of the displacement density probability of the water molecules, also known as Ensemble Average Propagator (EAP) [2, 13], it is possible to obtain a complex valued signal. The complex signal is generated, along one gradient direction, for a voxel located in the proximity of a 
single infinite plate. The noisy complex and magnitude signals are then obtained and the reconstruction of the magnitude diffusion signal is performed from each of them. The reconstructed signal, as the linear combination of basis functions, leads to an effective characterization of the diffusion properties and is a useful tool for measuring noise-related performances. In this respect we employed the Simple Harmonic Oscillator based Reconstruction and Estimation (SHORE) [8]. Within this framework, a Maximum Likelihood Estimator (MLE), for the reconstruction based on the Rician magnitude signal, is also provided for performance comparison.

\section{Methods}

In this section we present the theoretical framework. First, we present the diffusion signal equation for a voxel in a position close to a single infinite plate. Then we recapitulate the SHORE formulation for the signal fitting. Finally we describe the integration of the MLE in the SHORE.

\subsection{Signal in the proximity of a single infinite plate}

When considering a voxel located in the proximity of a restricting boundary such as an infinite plate, with voxel's dimensions significantly smaller than the separation distance between the plate and any other boundary, the magnetization in the voxel is influenced only by the boundary in the vicinity [9]. A graphical representation of this scenario is shown in Fig. $1 a$, where an infinite plate is represented with its normal aligned with the z-axis, and a voxel with height $z_{2}-z_{1}$ is located at a distance $z_{1}$ from the plate itself. Using the notation in [9] it is convenient to define the dimensionless position variable

$$
\zeta=\frac{z}{u}
$$

where $u$ is the characteristic diffusion length given by

$$
u=\sqrt{4 D_{0} \Delta}
$$

with $D_{0}$ being the free diffusion coefficient and $\Delta$ the diffusion time. Fig. $1 a$ also shows the gradient vector which, in this case, is assumed to be aligned with the positive z-axis. The gradient vector magnitude is then given by $q=\gamma \delta G / 2 \pi$ where $\gamma$ is the gyromagnetic ratio, $\delta$ is the diffusion pulse duration and $G$ is the diffusion gradient strength. The considered experiment is the pulsed gradient spin echo (PGSE) sequence with pulse duration $\delta$ small compared to $\Delta$. Finally, after defining the dimensionless wave-number $\kappa=\pi q u$, the complex signal is given by [9] 

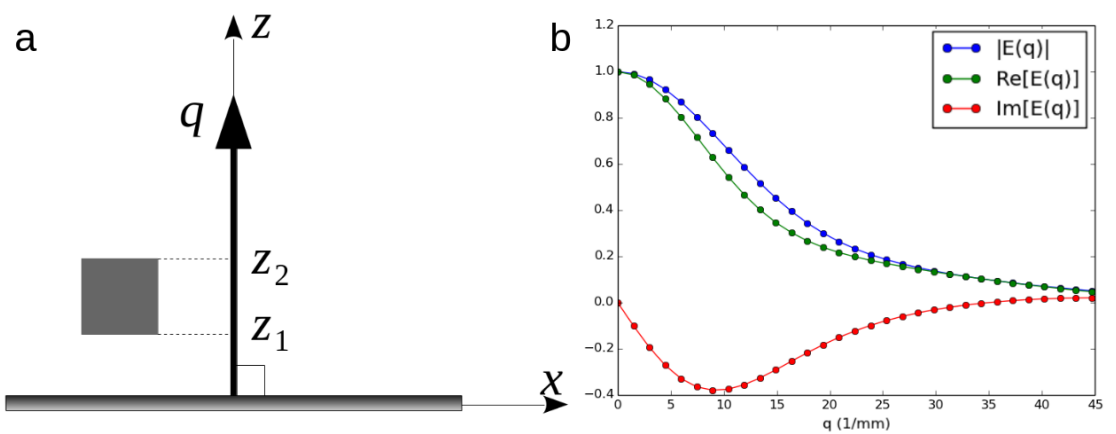

Fig. 1 (a) An infinite plate lies along the x-axis, with normal along the z-axis. A voxel is located at a distance $z_{1}$ from the infinite plate and has a height given by $z_{2}-z_{1}$. The gradient vector $\mathbf{q}$ makes a right angle with the $x$-axis toward the positive z-axis; adapted from [9]. (b) Magnitude, real and imaginary parts of the signal generated according to Eq. 3 for a voxel with $z_{1}=0$ and dimensionless height of $0.25 ; \delta=3 \mathrm{~ms}, \Delta=100 \mathrm{~ms}, D_{0}=2.299 \times 10^{-9} \mathrm{~m}^{2} / \mathrm{s}$ and $G_{\max }=350 \mathrm{mT} / \mathrm{m}$.

$$
E\left(\left[\zeta_{1}, \zeta_{2}\right], \kappa\right)=e^{-\kappa^{2}} \frac{F\left(\zeta_{2}\right)-F\left(\zeta_{1}\right)}{2\left(\zeta_{2}-\zeta_{1}\right)}
$$

where $\zeta_{1}$ and $\zeta_{2}$ are the dimensionless coordinates of the voxel corresponding to $z_{1}$ and $z_{2}$ via Eq. 1 , and $F(\zeta)$ is

$$
\begin{aligned}
F(\zeta)= & \zeta+\frac{1}{\sqrt{\pi}} e^{-(\zeta-i \kappa)^{2}}+\frac{e^{i 4 \kappa \zeta}}{i 4 \kappa}[1-\operatorname{erf}(\zeta+i \kappa)] \\
& -\left[\zeta-i\left(\kappa+\frac{1}{4 \kappa}\right)\right] \operatorname{erf}(-\zeta+i \kappa)
\end{aligned}
$$

\subsection{Signal reconstruction}

The signal fitting is performed with SHORE [8], a promising signal reconstruction method suitable for q-space magnetic resonance. Within this framework the signal is represented as the linear combination of orthogonal basis functions, result of the multiplication between an exponential and an Hermite polynomial

$$
\phi_{n}(u, q)=i^{-n} \sqrt{\frac{u \sqrt{\pi}}{2^{n-2} n !}} e^{-2 \pi^{2} q^{2} u^{2}} H_{n}(2 \pi q u)
$$

where $n$ is the order of the basis, $H_{n}(x)$ is the $n$ th-order Hermite polynomial and $u$ is the characteristic data dependent length or scaling factor to be determined, for instance by fitting the signal to an exponential decay according to $E(q)=$ $\exp \left(-2 \pi^{2} q^{2} u^{2}\right)$. Our formulation of the basis functions in Eq. 5 differs from the one given in [8] with the introduction of the normalizing factor $2 \sqrt{u \sqrt{\pi}}$, which 
renders the bases orthonormal. The bases are well suited for representing the signal in the complex domain: the even order basis functions are real valued and evenly symmetric whereas the odd order basis functions are imaginary and show odd symmetry, which is precisely the case of the real and the imaginary parts of the diffusion signal. The normalized diffusion signal in the SHORE representation can thus be expressed by

$$
E(q)=\sum_{n=0}^{N-1} a_{n} \phi_{n}(u, q)
$$

where $N$ is the maximum allowed order in the reconstruction and $a_{n}$ are the coefficients corresponding to the respective bases. The choice of $N$ directly affects the signal reconstruction: in the case of noisy data, a high order will potentially cause the reconstruction to follow the noise, whereas a low order will inherently enforce a smoothing effect. For a given order $N$ the signal reconstruction in the complex domain is performed by considering the even and odd coefficients for the real and imaginary parts respectively. In the case of the magnitude signal reconstruction, only the even coefficients are taken into account. In any case, a fitting procedure with a Linear Least Squares approach is used to estimate the coefficients.

\section{Maximum likelihood estimation}

To better take into account the Rician distribution of the noise affecting the diffusion magnitude signal, the estimation of the coefficients can be performed with the Maximum Likelihood Estimator (MLE). In the case of Rician noise, the MLE has been introduced in [12]. Normally several noisy realizations of each signal sample are required to properly estimate via the MLE. However in the case of one noisy realization per sample, the signal samples can be interpreted as noisy realizations of a function, thus the likelihood is given by

$$
L=\prod_{i=1}^{n} \frac{M_{i}}{\sigma^{2}} e^{-\frac{M_{i}^{2}+[A c]_{i}^{2}}{2 \sigma^{2}}} \mathrm{I}_{0}\left(\frac{[A c]_{i} M_{i}}{\sigma^{2}}\right)
$$

where $n$ is the number of samples of the magnitude signal, $M_{i}$ is the $i$-th sample, $\sigma^{2}$ is the noise variance, $A$ is the real SHORE design matrix, $c$ is the even coefficients vector and $\mathrm{I}_{0}$ is the modified zeroth order Bessel function of the first kind. The MLE is then defined as the estimator maximizing $L$ or equivalently $\log L$

$$
\hat{c}_{M L}=\underset{c}{\operatorname{argmax}}(\log L) .
$$

The performance of the estimator is expected to increase with the increasing number of samples of the signal. It should then be noticed that the MLE requires $\sigma^{2}$ to be given. Thus a prior estimation of the signal noise variance is necessary. 


\section{Experimental results}

This section describes the parameters adopted for the generation of the complex signal (see Sect. 2.1) and how from this the noisy complex and magnitude signals are obtained. Then, the fitting procedures for the magnitude signal reconstruction based on the SHORE, in the complex domain and for the magnitude (with both LLS and MLE), are discussed. Finally we present the way the performances of the magnitude signal reconstructions are compared.

We generated the complex diffusion signal, according to Eq. 3, for a voxel adjacent to an infinite plate and with dimensionless height of 0.25 (Fig. 1b). A total of $n$ equally spaced samples of the signal, with $n \in\{1,2, \ldots, 100\}$, is generated along the direction normal to the infinite plate (Fig. $1 a$ ). The sequence parameters are fixed to $G_{\max }=350 \mathrm{mT} / \mathrm{m}, \delta=3 \mathrm{~ms}, \Delta=100 \mathrm{~ms}$ and the physical quantities to $\gamma=2.675 \times 10^{8} \mathrm{rad} / \mathrm{s} T$ and $D_{0}=2.299 \times 10^{-9} \mathrm{~m}^{2} / \mathrm{s}$. Uncorrelated Gaussian noise with equal variance is added on the real and imaginary parts of the signal, obtaining the noisy complex signal. From this the magnitude is computed, obtaining the noisy magnitude signal. The standard deviation of the noise is calculated for several different SNR values by $\sigma=S N R^{-1}$ relatively to the non-weighted signal sample $(G=0)$.

The noisy complex and magnitude signals are then fitted via SHORE according to Eq. 6. The even (real) and odd (imaginary) SHORE bases are used for the complex signal fitting whereas only the even bases are used in the case of the magnitude signal fitting. Moreover the magnitude signal is fitted also with the MLE, obtaining the vector of the SHORE coefficients according to Eq. 8. To observe the influence of the maximum allowed order in the SHORE reconstruction $N$ (see Sect. 2.2 and Eq. 6), two orders are tested: one relatively low order $N=6$ and one relatively high order $N=10$. However the following discussion is referred to $N=6$ and a comparison with $N=10$ will be given later in Sect. 4 . In order to render the fitting of the complex and magnitude signals independent from the estimation of the scaling factor $u$, in every case the $u$ estimated on the noisy magnitude is also used in the complex fitting.

After the complex based fitting a real and an imaginary vectors of coefficients are obtained, leading to a real and an imaginary reconstruction respectively. These reconstructions are then used to compute the magnitude reconstruction from the complex fitting $\left(\mathscr{M}_{C}\right)$, which is then compared to the one reconstructed from the noisy magnitude signal via both $\operatorname{LLS}\left(\mathscr{M}_{M}\right)$ and MLE $\left(\mathscr{M}_{M_{M L}}\right)$ based fittings.

We performed the comparison by calculating the Root Sum of Squares (RSS) value of the residuals between the magnitude reconstructions $\left(\mathscr{M}_{C}, \mathscr{M}_{M}, \mathscr{M}_{M_{M L}}\right)$ and the ground truth $\mathscr{M}_{G T}=|E(q)|$. The whole procedure is performed with 1000 different noise realizations for each couple of SNR and number of samples $n$ (sampling resolution). Hence a triple of averaged RSS values, $R S S_{C} \pm S D_{R S S_{C}}, R S S_{M} \pm S D_{R S S_{M}}$ and $R S S_{M_{M L}} \pm S D_{R S S_{M_{M L}}}\left(R S S_{M L}, S D_{R S S_{M L}}\right.$ from now on and in the figures) is obtained for each pair $(S N R, n)$. For each value of $n$, the RSS values and their standard deviations can be represented as functions of the SNR, obtaining the curves $\{R S S\}$ and $\left\{S D_{R S S}\right\}$ as shown in Fig. $2 a$ and $2 b$ respectively $(n=31)$. 

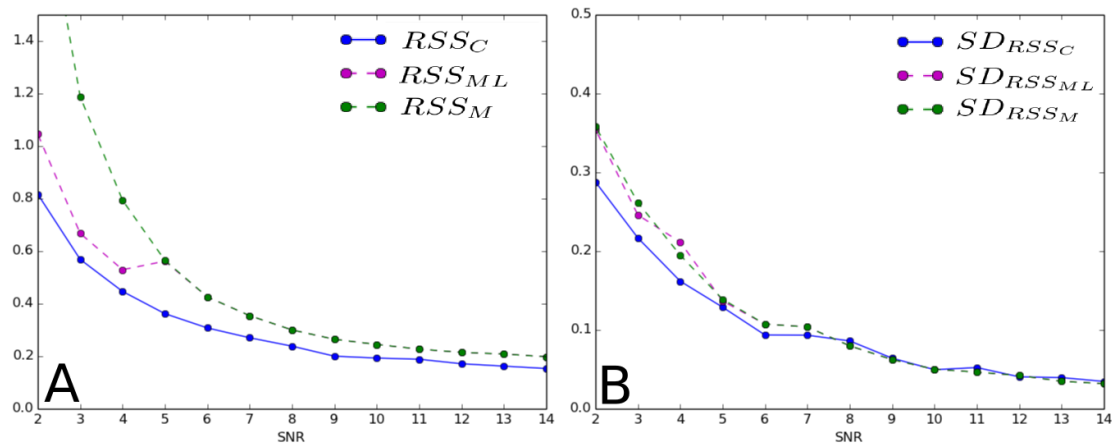

Fig. 2 Root sum of squares values $R S S_{C}, R S S_{M}$ and $\operatorname{RSS}_{M L}(a)$, and standard deviations $S D_{R S S_{C}}$, $S D_{R S S_{M}}$ and $S D_{R S S_{M L}}(b)$, as functions of the SNR. Each point represents the averaged value over 1000 noise realizations with the corresponding SNR. $G_{\max }=350 \mathrm{mT} / \mathrm{m}$, with $n=31$.

We also compared the reconstruction performances as function of the signal sampling resolution, i.e. as function of the number of samples $n$. Fig. 3 shows the integral of the difference between the $R S S$ (light blue) and $S D_{R S S}$ (magenta) curves of different techniques, as function of the number of samples $n$. Precisely, it shows the integral values of $\left\{R S S_{M}\right\}-\left\{R S S_{C}\right\}$ and $\left\{S D_{R S S_{M}}\right\}-\left\{S D_{R S S_{C}}\right\}$ (Fig. $\left.3 a\right),\left\{R S S_{M L}\right\}-$ $\left\{R S S_{C}\right\}$ and $\left\{S D_{R S S_{M L}}\right\}-\left\{S D_{R S S_{C}}\right\}$ (Fig. $3 b$ ), and finally $\left\{R S S_{M}\right\}-\left\{R S S_{M L}\right\}$ and $\left\{S D_{R S S_{M}}\right\}-\left\{S D_{R S S_{M L}}\right\}$ (Fig. $3 c$ ). Thus a positive value globally indicates a better reconstruction (less overall reconstruction error) for $\mathscr{M}_{C}$ compared to $\mathscr{M}_{M}$ (Fig. $3 a$ ), $\mathscr{M}_{C}$ compared to $\mathscr{M}_{M_{M L}}$ (Fig. $3 b$ ) and $\mathscr{M}_{M_{M L}}$ compared to $\mathscr{M}_{M}$ (Fig. $3 c$ ). It should be noticed that Fig. $3 c$ substantially describes the difference between Fig. $3 a$ and $3 b$.

Figure 4 shows the inter-technique comparisons of the RSS values $(a, b, c)$ and their standard deviations $(d, e, f)$, as function of both the SNR and the sampling resolution (i.e. $n$ ). In detail it shows the differences $R S S_{M}-R S S_{C}$ (Fig. $4 a$ ) and $S D_{R S S_{M}}-$ $S D_{R S S_{C}}$ (Fig. $4 d$ ), $R S S_{M L}-R S S_{C}$ (Fig. $4 b$ ) and $S D_{R S S_{M L}}-S D_{R S S_{C}}$ (Fig. 4e), and finally $R S S_{M}-R S S_{M L}$ (Fig. $4 c$ ) and $S D_{R S S_{M}}-S D_{R S S_{M L}}$ (Fig. $4 f$ ). Thus a positive value (red) indicates a better reconstruction $\mathscr{M}_{C}$ compared to $\mathscr{M}_{M}$ (Fig. 4a,d), $\mathscr{M}_{C}$ compared to $\mathscr{M}_{M_{M L}}$ (Fig. $4 b, e$ ) and $\mathscr{M}_{M_{M L}}$ compared to $\mathscr{M}_{M}$ (Fig. 4c,f). It should be noticed that the range of values in the color bars are different. For instance the amplitude of the range of the performance gain for the complex reconstruction, compared to the magnitude based one, is higher when the comparison is made with respect to the LLS fitting (Fig. 4a) than when the MLE is considered (Fig. 4b). The opposite holds for the performance loss. On the contrary we observe that the range of the performance gain/loss in the standard deviation is higher when the comparison refers to the MLE (Fig. 4e) rather than the LLS (Fig. $4 d$ ). Finally Fig. 5 shows the map of the best reconstruction technique for each pair of $S N R$ and $n$, that is the technique giving less reconstruction error (RSS value). In order to show the influence of the maximum reconstruction order of SHORE $N$ on the results, maps are generated for $N=6$ (Fig. $5 a$ ) and $N=10$ (Fig. $5 b$ ). 


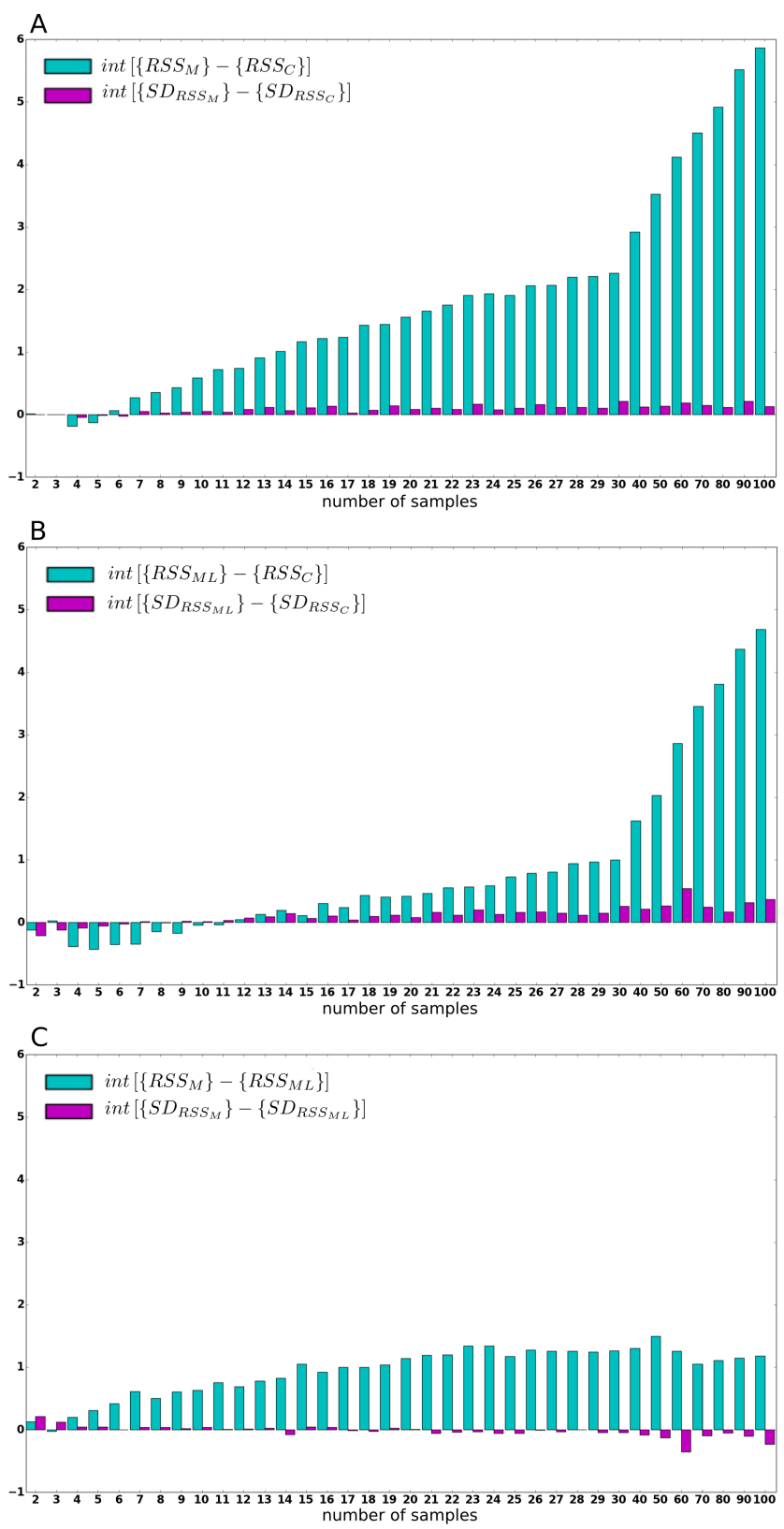

Fig. 3 In light blue the differences between the areas under the RSS curves of two different compared techniques (i.e. the curves in Fig. 2a); in magenta the differences between the areas under the $S D_{R S S}$ curves (i.e. the curves in Fig. $2 b$ ). Values are shown as a function of the number of samples $n$. A positive value indicates a globally better reconstruction (less overall reconstruction error) for $\mathscr{M}_{C}$ over $\mathscr{M}_{M}(a), \mathscr{M}_{C}$ over $\mathscr{M}_{M_{M L}}(b)$ and $\mathscr{M}_{M_{M L}}$ over $\mathscr{M}_{M}(c)$. $G_{m a x}=350 \mathrm{mT} / \mathrm{m}$. 

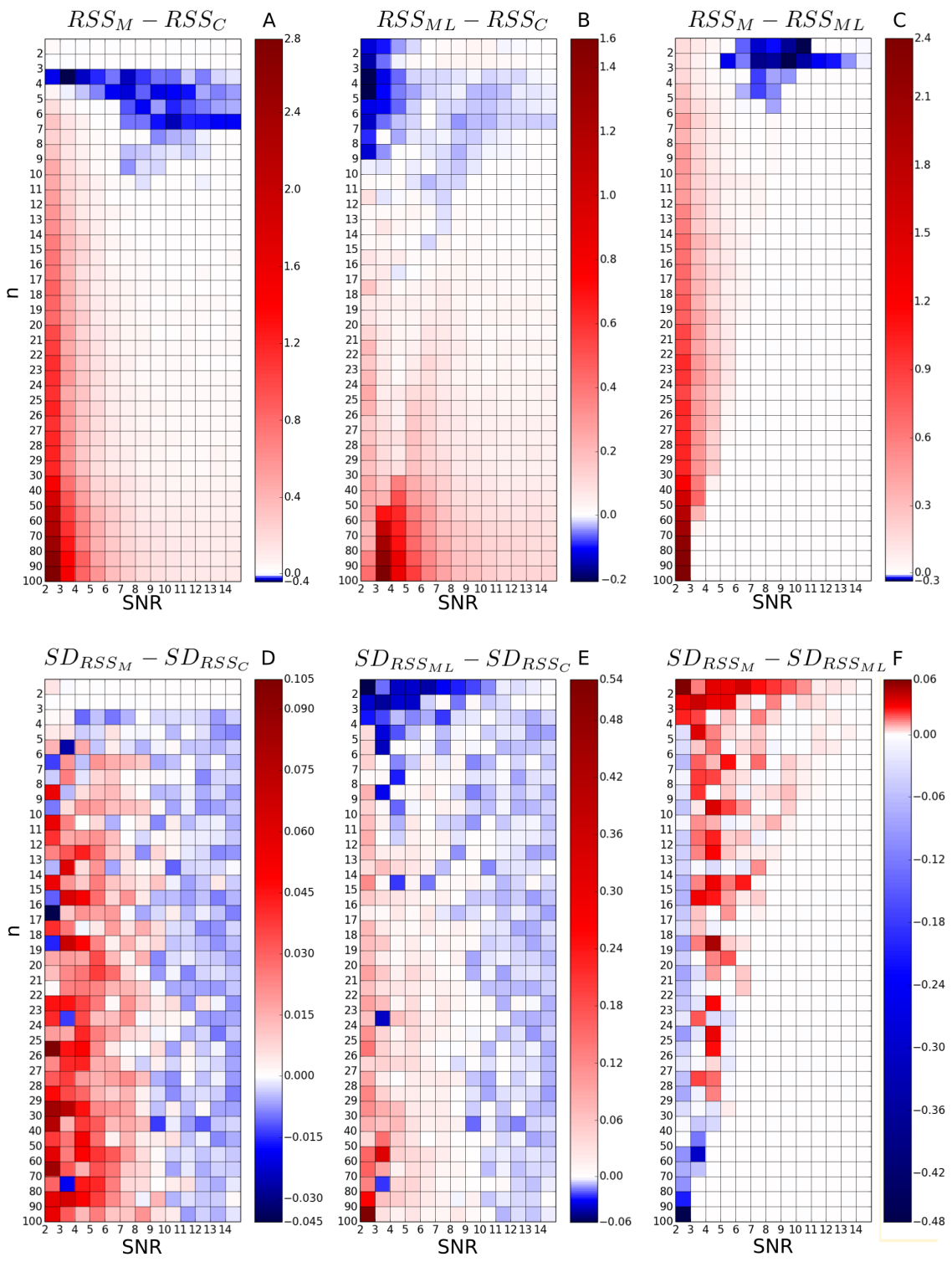

Fig. 4 Differences between $R S S$ values $(a, b, c)$ and correspondent $S D_{R S S}(d, e, f)$ for different compared techniques, as function of the SNR and of the number of samples $n$. A positive value (red) indicates a better reconstruction for $\mathscr{M}_{C}$ compared to $\mathscr{M}_{M}(a), \mathscr{M}_{C}$ compared to $\mathscr{M}_{M_{M L}}$ (b) and $\mathscr{M}_{M_{M L}}$ compared to $\mathscr{M}_{M}(c)$, or less error variance $(d, e, f)$. Each pixel represents the average value over 1000 noise realizations for the corresponding SNR and number of samples $n$. $G_{\text {max }}=350 \mathrm{mT} / \mathrm{m}$. 

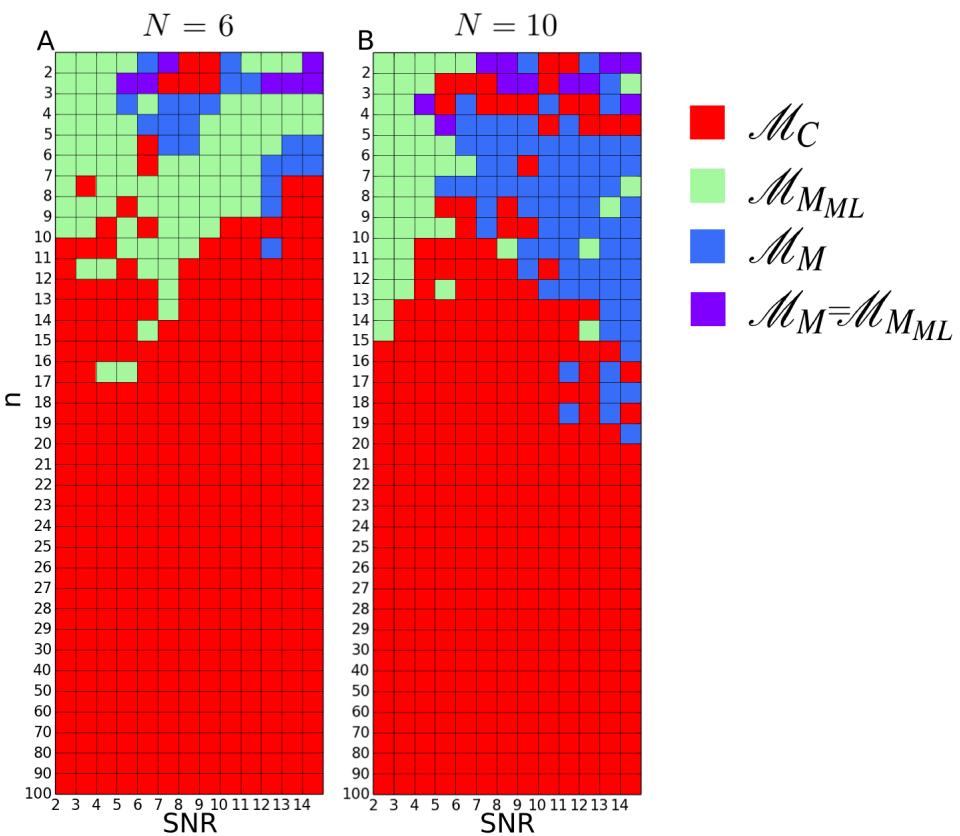

Fig. 5 The best reconstruction technique as a function of the SNR and the number of samples $n$, for a maximum reconstruction order $N=6(a)$ and $N=10(b)$, see Sect. 2.2 and Eq. 6 for reference. Red, green and blue colors indicate that the signal reconstruction showing less error is the one performed by fitting the noisy complex signal, $\mathscr{M}_{C}$, the noisy magnitude signal with the Maximum Likelihood Estimator (MLE), $\mathscr{M}_{M_{M L}}$, and with Linear Least Squares (LLS), $\mathscr{M}_{M}$, respectively. A purple color indicates that the best reconstruction is based on the noisy magnitude signal fitting, without any preference regarding the estimation technique.

We performed the reconstructions also for a signal generated with a set of parameters achievable in experimental conditions. In detail the voxel size is set to $50 \mu m, G_{\max }=60 \mathrm{mT} / \mathrm{m}, \delta=15 \mathrm{~ms}$ and $\Delta=50 \mathrm{~ms}$. Results are shown in Fig. 6 for $N=6$. More precisely it shows the comparison between the RSS value of the complex based reconstruction and that of the magnitude based reconstruction for a LLS approach Fig. $6 a$ and for a MLE apporach Fig. $6 b$. The range of SNR values $[2,20]$ in the figures is set to that showing major differences (values different from zero) in the results and corresponds to that generally adopted [7]. The number of samples $n$ range has been focused to $[2,15]$, in fact at higher values the trend is similar to that shown in Fig. 5. Figure $6 c$ finally shows the best reconstruction technique for SNR values up to 50 and is representative also for higher SNR up to 100 . 

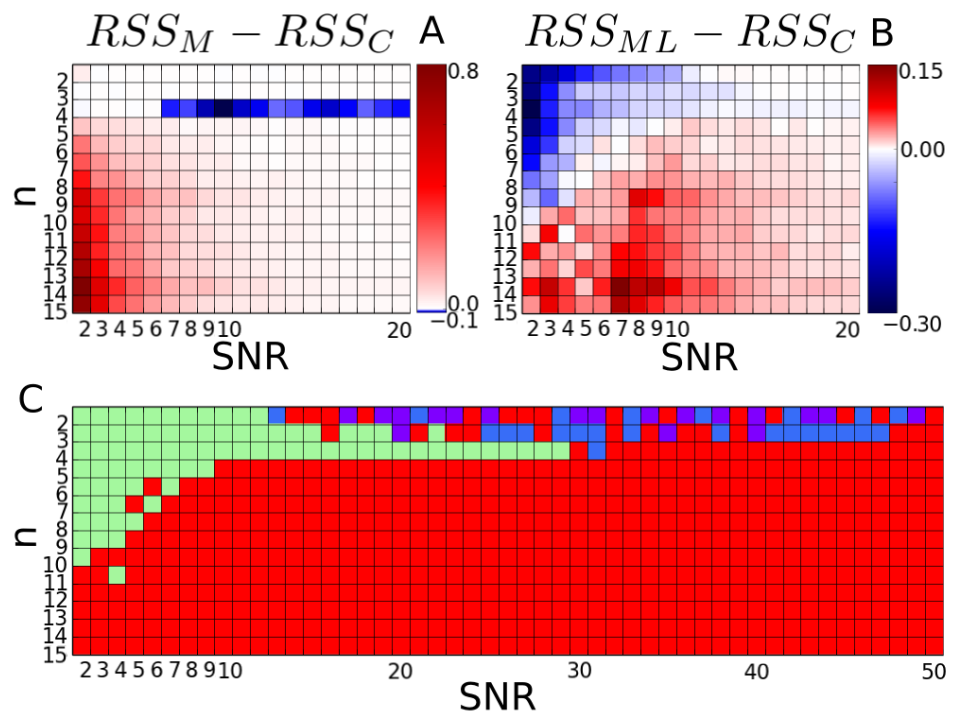

Fig. 6 Differences between RSS values for different compared techniques $(a, b)$ and best reconstruction technique as function of $n$ and $S N R(c)$. Results reported for a voxel with side $50 \mu \mathrm{m}$ and with $G_{\max }=60 \mathrm{mT} / \mathrm{m}, \delta=15 \mathrm{~ms}, \Delta=50 \mathrm{~ms}$. A positive value (red) indicates a better reconstruction for $\mathscr{M}_{C}$ compared to $\mathscr{M}_{M}(a)$ and for $\mathscr{M}_{C}$ compared to $\mathscr{M}_{M_{M L}}(b)$. (c) The best reconstruction technique is given for each pair $(n, S N R)$. Red, green and blue colors indicate that the signal reconstruction showing less error is the one performed by fitting the noisy complex signal, $\mathscr{M}_{C}$, the noisy magnitude signal with the Maximum Likelihood Estimator (MLE), $\mathscr{M}_{M_{M L}}$, and with Linear Least Squares (LLS), $\mathscr{M}_{M}$, respectively. A purple color indicates that the best reconstruction is based on the noisy magnitude signal fitting, without any preference regarding the estimation technique.

\section{Discussion of the results}

The noisy complex and magnitude signals have been fitted with the SHORE obtaining the complex $\mathscr{M}_{C}$ and the magnitude based $\mathscr{M}_{M}$ reconstructions respectively. The noisy magnitude has also been reconstructed by using the MLE, $\mathscr{M}_{M_{M L}}$. In each of the three cases $\mathscr{M}_{C}, \mathscr{M}_{M_{M L}}$ and $\mathscr{M}_{M}$ the reconstruction error has been calculated and compared as function of the SNR and the number of samples $n$.

The magnitude reconstruction performed by fitting the complex domain signal, $\mathscr{M}_{C}$, globally shows a better performance (less reconstruction error) compared to the one based on the LLS fitting on the magnitude, $\mathscr{M}_{M}$, for $n>5$ (Fig. $3 a$ ). The performance gain for the complex reconstruction is particularly evident for $S N R \leq$ 5 (Fig. 4a), as expected. Indeed at higher SNR values the Rician distribution is well approximated by a Gaussian [10,11], thus the performances of magnitude and complex based reconstructions should be equivalent. However an opposite trend is registered in case of $n=4,5$.

Moreover a performance dependence with the sampling resolution is observed. In first place, by increasing the sampling resolution $(n)$ the complex reconstruction 
$\mathscr{M}_{C}$ shows less error compared to the magnitude based one $\mathscr{M}_{M}$ also at $S N R>5$; with this regard the range of SNR values in which the performance gain is observed also increases (Fig. 4a). Secondly, the amount of error reduction in the case of $\mathscr{M}_{C}$ with respect to $\mathscr{M}_{M}$ follows the same trend.

Similar observations apply when the complex based reconstruction $\mathscr{M}_{C}$ is compared to that obtained via MLE based fitting of the magnitude $\mathscr{M}_{M_{M L}}$, but in this case for $n>11$ (Fig. 3b). Indeed the MLE for the magnitude based fitting, slightly improves the overall performance with respect to LLS at any sampling resolution for low SNR values (Fig. 3c). However it should be noticed that the MLE requires an estimation of the noise variance, and in the present case the exact value was supplied. The reconstruction improvement given by the MLE over the LLS also happens for $S N R<5$ (Fig. $4 c$ ), as clearly represented in the example of Fig. $2 a$. In addition the amplitude of the error reduction obtained with MLE over LLS increases with the sampling resolution $(n)$, whereas the range of SNR within which a performance gain is observed reduces (Fig. 4c).

Moreover, a performance loss for the MLE on the magnitude when compared to the LLS is observed within a region with $n \leq 6$ and $S N R \in[6,13]$ at different grades (Fig. 4c). However the limits of this region seem to be dependent on the maximum reconstruction order $N$ allowed in the SHORE, as shown in Fig. $5 a$ for $N=6$ and $5 b$ for $N=10$. In fact when $N=10$ the range of the SNR values within which the performance gain of the MLE over the LLS is observed reduces. On the other hand the range of the number of samples $n$ within which $\mathscr{M}_{M_{M L}}$ shows less error than $\mathscr{M}_{C}$, at very low SNR, slightly increases. Otherwise, the region in which the complex based reconstruction is the most performing do not seems to be affected by the choice of $N$.

Finally the complex based fitting leads to a reconstruction with an error standard deviation $S D_{R S S_{C}}$ lower than $S D_{R S S_{M}}$ for $n>6$ (Fig. $3 a$ and $4 d$ ). Similar observations apply when $S D_{R S S_{C}}$ is compared to $S D_{R S S_{M L}}$ for $n>8$ (Fig. 3b), although at low SNR the MLE shows a lower standard deviation (Fig. $4 e$ and $4 f$ ).

Very similar considerations apply when the reconstructions are performed and evaluated in the case of signal generated with different voxel and pulse sequence parameters as shown in Fig. 6. However in this case the region in which the magnitude based reconstruction shows better performances (Fig. $6 c$ ) is almost entirely defined by the performance of the MLE (Fig. $6 b$ ). Indeed almost everywhere in this region the performance of the LLS is comparable or inferior to that of the complex based reconstruction (Fig. $6 a$ ). Finally at higher SNR values the complex based reconstruction is generally the one showing the best performance (Fig. $6 c$ ).

Hence, as shown in Fig. $3 b, 5 a$ and $6 c$, at low SNR values it might be better to perform a reconstruction on the noisy magnitude signal when $n \leq 10$ with order $N=6$ and $n \leq 15$ with order $N=10$ (Fig. $5 b$ ). Within this region the MLE generally shows better performances for $S N R \leq 5$. However in the rest of the cases a reconstruction performed in the complex domain generally leads to less reconstruction error. 


\section{Conclusions}

We have presented a comparative analysis between the diffusion signal reconstruction on the magnitude and in the complex domain. For the magnitude signal reconstruction we relied on the SHORE bases for both the noisy complex and magnitude fittings. In the case of the noisy (Rice distributed) magnitude fitting we introduced the maximum likelihood based (MLE) coefficients estimation for the SHORE, as an alternative to the standard Linear Least Squares based approach (LLS). We compared the performance of the reconstructions as a function of the SNR value and the number of samples in the signal $n$ (sampling resolution).

Our results show that the choice of which reconstruction technique to adopt mainly depends on the SNR and on the number of samples of the signal, as shown in Fig. 5a,b and Fig. $6 c$. Depending on these parameters, the best magnitude signal reconstruction can be achieved for any of the compared techniques. However the complex based reconstruction reveals to be the most performing at any SNR with the increasing total number of samples of the diffusion signal. To further improve its performance, regularization constraints could also be implemented. This would be beneficial for the estimation of the diffusion parameters.

Despite the difficulty that may be encountered in clinical conditions in achieving an acquisition setup such as the one here adopted, the presented results might be directly useful and potentially validated in experimental setups.

\section{Acknowledgments}

The authors would like to express their thanks to Olea Medical and to the ProvenceAlpes-Côte d'Azur (P.A.C.A.) Regional Council for providing grant and support for this work. The authors also thank Demian Wassermann for the careful reading.

\section{References}

1. Basser, P. J.: Relationships between diffusion tensor and q-space MRI. Magnetic resonance in medicine, 47(2), 392-397 (2002).

2. Callaghan, P. T., MacGowan, D., Packer, K. J., Zelaya, F. O.: High-resolution q-space imaging in porous structures. Journal of Magnetic Resonance (1969), 90(1), 177-182 (1990).

3. Constantinides, C. D., Atalar, E., McVeigh, E. R.: Signal-to-noise measurements in magnitude images from NMR phased arrays. Magnetic Resonance in Medicine, 38(5), 852-857 (1997).

4. Dietrich, O., Heiland, S., Sartor, K.: Noise correction for the exact determination of apparent diffusion coefficients at low SNR. Magnetic resonance in medicine, 45(3), 448-453 (2001).

5. Gudbjartsson, H., Patz, S.: The Rician distribution of noisy MRI data. Magnetic Resonance in Medicine, 34(6), 910-914 (1995).

6. Henkelman, R. M.: Measurement of signal intensities in the presence of noise in MR images. Medical physics, 12(2), 232-233 (1985).

7. Jones, D. K., Basser, P. J.: 'Squashing peanuts and smashing pumpkins': How noise distorts diffusion weighted MR data. Magnetic Resonance in Medicine, 52(5), 979-993 (2004). 
8. Özarslan, E., Koay, C. G., Basser, P. J.: Simple harmonic oscillator based estimation and reconstruction for one-dimensional q-space MR. In Proc. Intl. Soc. Mag. Reson. Med (Vol. 16, p. 35) (2008).

9. Özarslan, E., Nevo, U., Basser, P. J.: Anisotropy induced by macroscopic boundaries: surfacenormal mapping using diffusion-weighted imaging. Biophysical journal, 94(7), 2809-2818 (2008).

10. Pajevic, S., Basser, P. J.: Parametric and non-parametric statistical analysis of DT-MRI data. Journal of magnetic resonance, 161(1), 1-14 (2003).

11. Salvador, R., Pena, A., Menon, D. K., Carpenter, T. A., Pickard, J. D., Bullmore, E. T.: Formal characterization and extension of the linearized diffusion tensor model. Human brain mapping, 24(2), 144-155 (2005).

12. Sijbers, J., den Dekker, A. J., Scheunders, P., Van Dyck, D.: Maximum-likelihood estimation of Rician distribution parameters. IEEE Trans. Med. Imaging, 17(3), 357-361 (1998).

13. Stejskal, E. O.: Use of Spin Echoes in a Pulsed Magnetic-Field Gradient to Study Anisotropic Restricted Diffusion and Flow. Journal of Chemical Physics, 43, 3597-3603 (1965).

14. Veraart, J., Sijbers, J., Sunaert, S., Leemans, A., Jeurissen, B.: Weighted linear least squares estimation of diffusion MRI parameters: Strengths, limitations, and pitfalls. NeuroImage, 81, 335-346 (2013). 